\title{
VACUNACIÓN EN PERSONAS CON DIABETES MELLITUS
}

\section{VACCINATION IN PEOPLE WITH DIABETES MELLITUS}

\author{
Cristian Suárez Cordo¹, Susana Salzberg¹, Claudia Folino¹, Pilar Cean¹, Julio César Bragagnolo', \\ Analía Dagum¹, Jorge Alvariñas ${ }^{1}$, Gustavo Frechtel' ${ }^{1}$, Alejandro Serra ${ }^{1}$
}

\section{RESUMEN}

Introducción: el Ministerio de Salud de la Nación (MSAL) recomienda la vacunación para proveer inmunidad a lo largo de la vida. A diferencia de lo que ocurre con la población infanto-juvenil, la mayoría de los adultos no se vacuna de acuerdo a las recomendaciones vigentes.

Objetivos: describir los esquemas de vacunación que deben recibir las personas con diabetes mellitus.

La vacuna antigripal debe administrarse en forma anual desde los 2 a los 64 años de edad.

Con respecto a la vacunación antineumocócica, existen dos vacunas disponibles: la VPN23 de 23 serotipos y la vacuna conjugada de 13 serotipos (VCN13). Entre los 5 a 64 años de edad se indica una primera dosis con VCN13, una segunda dosis con VPN23 y un refuerzo con VPN23 a los 65 años.

Si bien en Argentina la vacuna para hepatitis B se incorporó al calendario de vacunación para todos los adultos, debemos tener en cuenta que las personas con diabetes tipo 1 y 2 tienen mayor prevalencia de hepatitis $\mathrm{B}$ que la población general.

Conclusiones: el médico que asiste a personas con diabetes, especialmente adultas, debe mantenerse actualizado al respecto y estar listo para actuar como agente directo en la recomendación de vacunas para esta población.

Palabras clave: vacunación, VPN23, VCN13.

\section{ABSTRACT}

Introduction: the recommendations of the Argentine Ministry of Health (MSAL) to provide immunization throughout the life is vaccination. Unlike the child and youth population, most of adults do not receive vaccination according to the current recommendations.

Objectives: to describe the immunization schemes that people with diabetes mellitus should receive.

The influenza virus vaccine should be administered annually from 2 to 64 years old.

As regards the pneumococcal vaccine, there are two vaccines available: the PVN23 with 23 serotypes and the conjugated pneumococcal vaccine with 13 serotypes (PCV13). The first dose of PCV13 is indicated between 5 and 64 years old, a second dose with PVN23 and a booster shot of PVN23 at 65 years old.

Although in Argentina the hepatitis $B$ virus vaccine was introduced in the immunization scheme for all adults, we should take into consideration that people with Diabetes type I and II have higher prevalence of hepatitis $B$ than the common population. Conclusions: the treating physician of people with diabetes, mainly adults, should be updated on the matter and be ready to act as direct agent to recommend immunization for this type of population.

Key words: vaccination, PVN23, PCV23.

Revista de la Sociedad Argentina de Diabetes 2017; Vol. 51 (58-62)
${ }^{1}$ Comité de Farmacología de la Sociedad Argentina de Diabetes

Contacto del autor: Cristian Suárez Cordo

E-mail: csuarezcordo@gmail.com

Correspondencia: Paraguay 1307, piso $8^{\circ}$ ofic. 74

(C1057AAU), CABA, Argentina

Tel: (011) 11-5938-9511

Fecha de trabajo recibido: 08/06/17

Fecha de trabajo aceptado: 28/06/17

Conflictos de interés: Cristián Suárez Cordo: participación en disertaciones para Eli Lilly y Boehringer Ingelheim.

Susana Salzberg: participación en disertaciones para Pfizer, AstraZeneca, Novo Nordisk; investigación en Eli Lilly, Sanofi, Merck.

Claudia Folino: asesoró a Boehringer Ingelheim, Gador y Genzyme.
Pilar Cean: recibió honorarios de AstraZeneca y actualmente de Boehringer Ingelheim.

Julio César Bragagnolo: recibió honorarios de consultoría disertación o entrenamiento de disertantes o apoyo por asistencia a congresos o equipamiento institucional por AstraZeneca, Denver Farma, Investi Farma, Janssen, Montpellier, Novartis, Novo Nordisk, Roche y Sanofi. Analía Dagum: sin conflictos de interés.

Jorge Alvariñas: recibió honorarios por conferencias, asesoramiento, consultoría, cursos de los laboratorios Sanofi, AstraZeneca, Merck, Novo Nordisk, Craveri, Roche. Gustavo Frechtel: recibió honorarios por conferencias, consultoría, investigación, asesoramiento a Sanofi Aventis, Eli Lilly, Merck, Novo Nordisk, AstraZeneca.

Alejandro Serra: asesor médico en Química Montpellier. 


\section{INTRODUCCIÓN}

El Ministerio de Salud de la Nación (MSAL) recomienda la vacunación desde el nacimiento hasta la edad avanzada para proveer inmunidad a lo largo de la vida. En nuestro país, el calendario de vacunación muestra altas tasas de cumplimiento en la población infanto-juvenil debido tanto a la eficacia asistencial de los médicos pediatras, que han abonado a la concientización a lo largo de varios años, como a la especial sensibilidad de la población frente a prevención en la niñez. En sentido contrario, según mencionan los registros oficiales, la mayoría de los adultos no se vacuna de acuerdo a las recomendaciones vigentes.

El médico que asiste a personas con diabetes debe tener presente los aspectos relevantes de la inmunización en esta población. Los niños y adultos con diabetes, como la población general, deben recibir el esquema de vacunación de acuerdo a las recomendaciones específicas según la edad determinadas por el MSAL 2017?.

Las vacunas recomendadas por el MSAL en las personas con diabetes se detallan a continuación:

\begin{tabular}{|c|c|c|c|c|}
\hline Años & Gripe & $\begin{array}{c}\text { Neumococo } \\
\text { VCN13 }\end{array}$ & $\begin{array}{c}\text { Neumococo } \\
\text { VPN23 }\end{array}$ & Hepatitis B \\
\hline $\begin{array}{c}6 \text { meses a } \\
5 \text { años }\end{array}$ & \multirow{3}{*}{ Anual } & \multicolumn{3}{|c|}{ Seguir calendario de vacunación obligatorio } \\
\hline $\begin{array}{l}5 \text { a } 64 \\
\text { años }\end{array}$ & & 1 dosis & 1 dosis & \multirow{2}{*}{$\begin{array}{c}\text { Iniciar o } \\
\text { completar } \\
\text { esquema } \\
3 \text { dosis } 0-1-6^{\text {meses }^{2}}\end{array}$} \\
\hline $\begin{array}{l}65 \text { ó } \\
\text { mayor }\end{array}$ & & \multicolumn{2}{|c|}{1 ó 2 dosis ${ }^{1}$} & \\
\hline
\end{tabular}

1) Ver recomendaciones en el párrafo de vacunación antineumocócica según grupo etario y vacunas recibidas previamente. 2) A partir de los 11 años de edad.

VCN13: vacuna conjugada; VPN23: vacuna con polisacáridos.

Fuente: MSAL 2017.

Figura 1: Esquema ilustrativo de la vacunación según edad y vacunas seleccionadas.

Las recomendaciones descriptas en la tabla no deben hacer olvidar el plan de vacunación habitual que forma parte del calendario nacional de vacunación obligatorio, por grupo etario o situación especial de riesgo como viajes, accidentes o contactos que se presentan en la población general.

\section{Vacuna antigripal}

El calendario nacional de vacunación incluye la vacuna antigripal anual para los grupos con mayor riesgo, y está disponible de manera gratuita en vacunatorios, hospitales y centros de salud públicos de todo el país ${ }^{2}$. Las personas con diabetes, desde los 2 a los 
64 años de edad, forman parte del grupo de riesgo donde se considera la vacunación, y podrán recibir la vacuna presentando la orden médica ${ }^{2}$. Cabe acotar que entre los 6 meses y los 2 años de vida se encuentra en el calendario oficial de vacunación en forma universal y hasta los 8 años inclusive se requiere ajustar la dosis ${ }^{3}$.

Las personas con diabetes, a partir de los 9 años, deberán recibir anualmente una sola dosis de la vacuna antigripal ${ }^{3}$.

\section{Vacuna antineumococo}

El impacto de la enfermedad neumocócica en adultos, en particular la neumonía adquirida en la comunidad (NAC) así como las formas invasivas (bacteriemia, meningitis, etc.), provoca anualmente una importante carga en este grupo etario, especialmente en adultos mayores y en aquellos con ciertas comorbilidades ${ }^{4}$.

Respecto del esquema de vacunación antineumocócica del MSAL incluye a los adultos con enfermedades inmunológicas o crónicas, y las personas con diabetes forman parte del grupo de alto riesgo de padecer enfermedad invasiva ${ }^{5-6}$.

En la actualidad existen dos vacunas disponibles: la vacuna con polisacáridos (VPN23) que contiene polisacáridos purificados de 23 serotipos de neumococo y la vacuna conjugada de 13 serotipos (VCN13) aprobada por ANMAT en 2012 con mayor inmunogenicidad comparada con la VPN23 (>para 10 de los 12 serotipos comunes y no inferior para los otros serotipos comunes) ${ }^{6}$. La VCN13 forma parte del calendario nacional de nuestro país en niños y se administran dos dosis y un refuerzo a los 2, 4 y 12 meses $^{5-6}$.

En los lineamientos técnicos y en el manual del vacunador del MSAL 2017 se indica la vacunación contra el neumococo, con orden médica, para el grupo de personas de 5 a 64 años de edad que presentan factores de riesgo para desarrollar enfermedad neumocócica invasiva, así como a quienes tienen diabetes, según el siguiente esquema:
- $1^{\circ}$ dosis: VCN13.

- $2^{\circ}$ dosis: VPN23 con un intervalo mínimo de 12 meses luego de la VCN13.

- $1^{\circ}$ refuerzo: VPN23 a los 65 años (respetando intervalo mínimo de 5 años de la dosis anterior).

Si alguna persona con indicación de esquema secuencial (que presente una o más de las comorbilidades arriba descriptas) anteriormente recibió VPN23, deberán respetarse los siguientes intervalos mínimos según la edad para recibir VCN137:

- Menores de 18 años: 8 semanas.

- Mayores de 18 años: 12 meses.

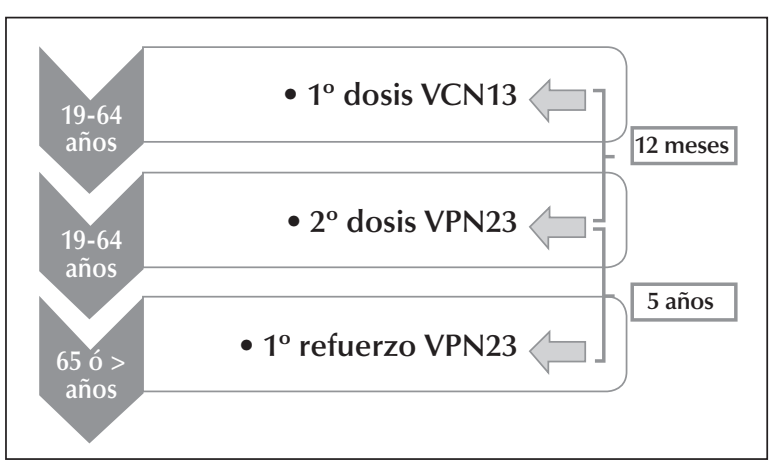

Figura 2: Esquema ilustrativo de vacunación antineumocócica?.

Requieren especial mención los adultos mayores con diabetes porque la prevalencia de la afección es creciente y compromete aproximadamente al $20 \%$ de la población. Se recomienda que ambas vacunas antineumocócicas -VCN13 y VPN23- se apliquen en serie en adultos de $\geq 65$ años. Con el objetivo de simplificar las recomendaciones de utilización de VCN13 y VPN23, se aconseja la armonización de los intervalos entre VCN13 y VPN23 independientemente del orden en el cual se administran en un plazo no menor a un año ${ }^{8}$.

En caso de seleccionar a pacientes no vacunados, se sugiere indicar primero una dosis de VCN13 y por lo menos un año después continuar con una dosis de VPN23. No deben coadministrarse. Si una dosis de VPN23 se administra inadvertidamente antes de cumplir el intervalo recomendado de un año, dicha dosis no debe repetirse. 


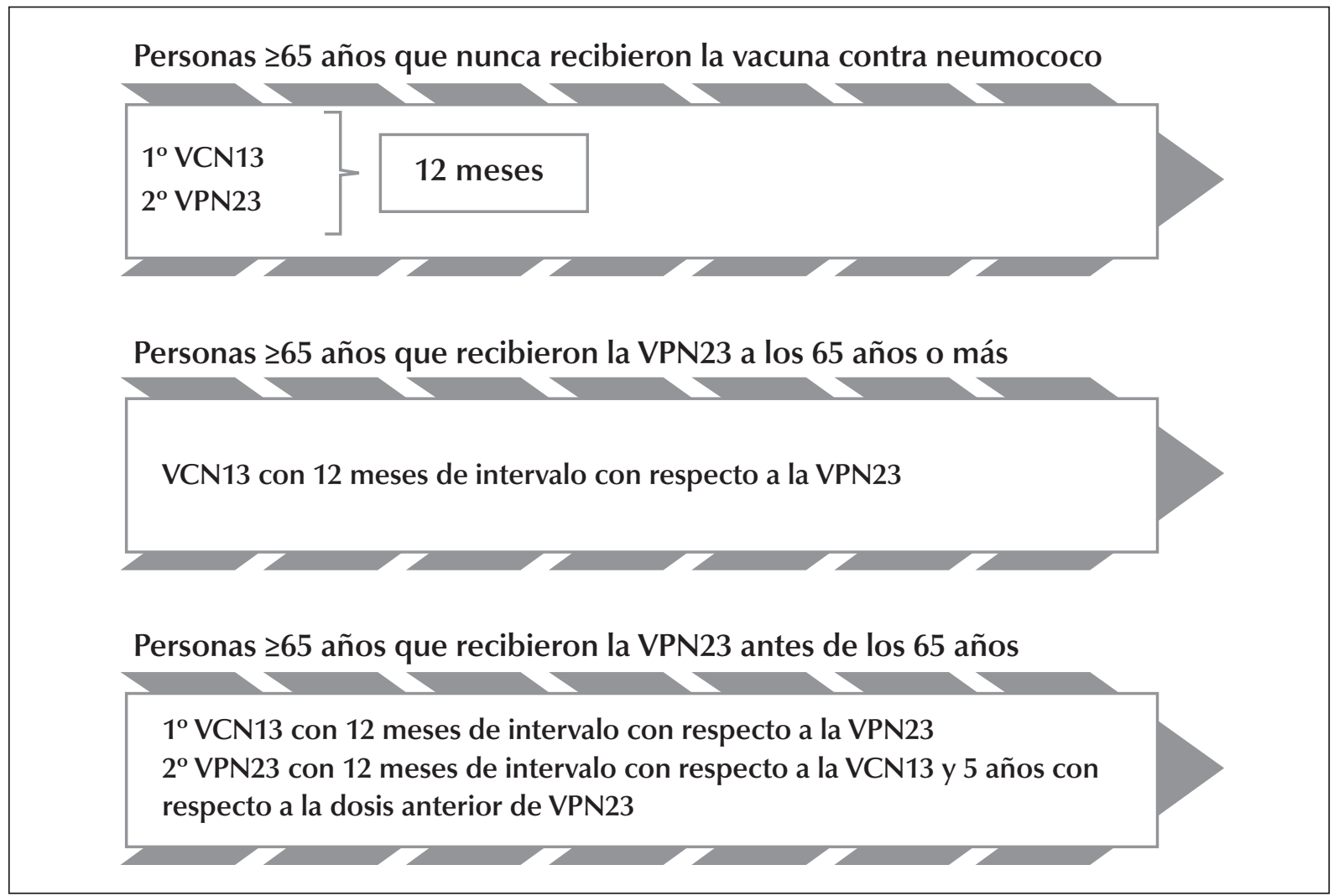

Figura 3: Esquema de vacunación en personas mayores de 65 años ${ }^{7-9}$.

\begin{tabular}{|c|c|c|c|c|}
\hline Vacuna & Nombre comercial & Dosis & Sitio & Detalles \\
\hline Antigripal $^{8}$ & $\begin{array}{l}\text { Viraflu } \\
\text { (Sanofi Pasteur) }\end{array}$ & $0,5 \mathrm{ml}$ & $\begin{array}{l}\text { IM-Deltoides } \\
\text { o Sc profunda }\end{array}$ & $\begin{array}{l}\text { Durante el otoño } \\
\text { (febrero a julio) }\end{array}$ \\
\hline \multirow[t]{3}{*}{ Antineumococo $^{7}$} & $\begin{array}{l}\text { Pneumo }{ }^{\circledR} 23 \\
\text { (Sanofi Pasteur) }\end{array}$ & $0,5 \mathrm{ml}$ & IM-Deltoides & \\
\hline & $\begin{array}{l}\text { Pneumovax }{ }^{\circledR} 23 \\
\text { (Merck Sharp Dhome) }\end{array}$ & $0,5 \mathrm{ml}$ & IM-Deltoides & \\
\hline & $\begin{array}{l}\text { Prevenar }^{\circledR} 13 \\
\text { (Pfizer) }\end{array}$ & $0,5 \mathrm{ml}$ & IM-Deltoides & \\
\hline
\end{tabular}

IM: intramuscular; Sc: subcutánea.

Tabla 1: Vacunas propuestas por el MSAL para $2017^{3-7}$.

\section{Vacuna hepatitis B}

Si bien en Argentina se incorporó al calendario de vacunación para todos los adultos, es importante resaltar la especial consideración en personas con diabetes.

Las personas con diabetes tipo 1 y 2 tienen mayor prevalencia de hepatitis $B$ que la población general. Dicha situación podría vincularse tanto por el contacto con sangre infectada, como por la falta de cumplimiento de normativas de bioseguridad en relación a accidentes con agujas, lancetas o descartables contaminados o por el uso inapropiado de equipos de automonitoreo glucémico tanto en condición ambulatoria como en internación $n^{10}$.

Otra circunstancia de especial interés en la inmunización contra la hepatitis b se relaciona con la presencia de insuficiencia renal 
en estadio 3 A (depuración de creatinina entre 60 y $45 \mathrm{~mL} / \mathrm{min} / 1,73 \mathrm{~m}^{2}$ ). En caso de no constar vacunación efectiva, se indica especialmente esta vacuna por la posibilidad de progresión a insuficiencia renal avanzada y la eventual necesidad de tratamiento sustitutivo renal.

\section{CONCLUSIONES}

La diabetes mellitus representa un problema de salud creciente y de afectación universal. Se encuentra en continua expansión epidemiológica y en estrecha relación con el incremento de la obesidad y el aumento de la edad de la población. La asistencia médica actual en los adultos reconoce y promueve un necesario vínculo entre los pacientes y sus médicos de cabecera, y varias veces los pacientes no los reconocen como referentes sobre las pautas de inmunización. Es así que la pérdida de oportunidades para prescribir las vacunas obliga sobre todo al diabetólogo de adultos a mantenerse actualizado al respecto y estar listo para actuar como agente directo en la recomendación de vacunas para sus pacientes.

\section{BIBLIOGRAFÍA}

1. Calendario Nacional de Vacunación. Ministerio de Salud de la Nación, Presidencia de la Nación, Argentina 2017. Disponible en: www.msal.gob.ar/images/stories/ryc/gra ficos/0000001013cnt-2017-01-01_calendario-vacunacion. pdf. Última consulta: 7 de mayo de 2017.

2. Ministerio de Salud. Cuidados en el invierno. Vacunación antigripal anual. Disponible en: www.msal.gov.ar/ cuidados-invierno/vacunacion-antigripal/. Última consulta: 7 de mayo de 2017.
3. Lineamientos técnicos. Manual del vacunador. Vacunación antigripal 2017. Disponible en: www.msal.gob.ar/ images/stories/bes/graficos/0000000950cnt-2017-04_ lineamientos-gripe_2017.pdf. Última consulta: 14 de mayo de 2017.

4. Sociedad Argentina de Infectología. Comisión Vacunas. Vacunas contra el neumococo en adultos. Agosto de 2015.

5. Ministerio de Salud. Cuidados en el invierno. Disponible en: www.msal.gob.ar/index.php/programas-yplanes/271-vacuna-neumococo. Última consulta: 7 de mayo de 2017.

6. Luna $\mathrm{CM}, \mathrm{y}$ col. Recomendaciones de vacunación en adultos con enfermedades respiratorias. Documento de la Asociación Argentina de Medicina Respiratoria para los Neumonólogos. Revista Americana de Medicina Respiratoria 2015; 15: 4. Disponible en: www.ramr. org/articulos/volumen_15_numero_4/articulos_especiales/articulos_especiales_recomendaciones_de_vacunacion_en_adultos_con_enfermedades_respiratorias. php\#fig2. Última consulta: 7 de mayo de 2017.

7. Lineamientos técnicos. Manual del vacunador. Vacunación contra neumococo 2017. Disponible en: www.msal. gob.ar/images/stories/bes/graficos/0000000947cnt-Lineamientos_neumo_adultos_WEB.pdf. Última consulta: 14 de mayo de 2017.

8. Use of 13-Valent Pneumococcal Conjugate Vaccine and 23-Valent Pneumococcal Polysaccharide Vaccine for adults with immunocompromising conditions: Recommendations of the Advisory Committee on Immunization Practices (ACIP) MMWR 2012; 61 (40): 816-819.

9. West nile virus and other nationally notifiable arboviral diseases. United States, 2014. MMWR 2015; 64 (34); 929:964.

10. Standards of Medical Care. Diabetes Care 2017 Jan; 40 (Supplement 1): S25-S32. https://doi.org/10.2337/dc17S006. 